\title{
Unusual Presentations of Actinomycosis; Anterior Abdominal Wall and Appendix: Report of Three Cases
}

\author{
Faruk Karateke, Sefa Özyazıcı, Ebru Menekşe, Koray Daş, Mehmet Özdoğan \\ Department of General Surgery, Numune Education and Research Hospital, Adana, Turkey
}

\begin{abstract}
Background: Primary actinomycosis of the anterior abdominal wall and appendix are very rare clinical entities. An accurate diagnosis is generally obtained by histological examination, and treatment often requires surgical resection.

Case Report: In this study we presented two cases of primary actinomycosis involving the anterior abdominal wall and a third one located in the appendix.

Conclusion: Actinomyces Israelii can involve all anatomic structures of the abdomen. Although preoperative diagnosis is difficult, the combination of surgery and antibiotic treatment results in complete treatment in the majority of cases.
\end{abstract}

Key Words: Actinomycosis, abdominal wall, appendix

Received: 21.09 .2012

Accepted: 14.11.2012

\section{Introduction}

Actinomyces israelii is a microaerophilic, anaerobic, grampositive, filamentous bacterium. It is normally found in humans and can colonise the respiratory, gastrointestinal and genitourinary tracts. Actinomycosis (AMC) is a subacute to chronic bacterial infection characterised by contiguous spread, suppurative and granulomatous inflammation. It typically causes the formation of multiple abscesses and sinus tracts. Actinomycosis leads most frequently cervicofacial (50\%), abdominal $(20 \%)$ and thoracic (15\%) involvements (1). Isolated abdominal wall disease is extremely rare, and to our knowledge, only 30 cases have been reported in the English literature to date (2). In this paper, we report two cases of actinomycosis of the anterior abdominal wall and a rare case of acute appendicitis caused by actinomycosis.

\section{Case Reports}

\section{Case 1}

A 30 year-old woman was admitted to our hospital with the complaint of a 2 week period of right upper quadrant swelling and pain. She had no history of weight loss, trauma, surgery, or intrauterine device. Upon admission, the physical exam revealed a non-movable mass in the right upper quadrant. She was febrile $\left(38.5^{\circ} \mathrm{C}\right)$. Blood investigations revealed haemoglobin of $13.1 \mathrm{~g} / \mathrm{dL}$ and white blood cell count (WBC) of $18,200 / \mathrm{mm}^{3}$. Serum amylase, blood electrolytes, liver function tests and chest radiography were normal. Computed tomography (CT) of the abdomen with oral and intravenous contrast identified an irregular density, measuring $5 \times 8 \mathrm{~cm}$ arising from the internal abdominal wall. The lesion showed a faint contrast enhancement and mild inflammatory changes with no involvement of the intestines (Figure 1a).

A right subcostal laparotomy revealed an indurated mass, projecting from the posterior sheath of the rectus abdominis, without invasion of the peritoneum and abdominal viscera (Figure $1 \mathrm{~b}$ ). Surgical excision of the inflammatory mass was performed and primary closure of the wound was carried out.

The histopathological examination revealed an acuteon-chronic extended abscess of the right abdominal recta muscle with heavy infiltration of chronic inflammatory cells and fibrous tissue with Gram-positive filamentous organisms (Actinomyces with typical sulphur granules) (Figure 1c). The patient was commenced on intravenous penicillin G (20 million IU/day) for 4 weeks postoperatively and treatment was prolonged with peroral application of amoxicillin at a dose of $2 \mathrm{~g} /$ day over the following 1 -month period.

\section{Case 2}

The patient was a 34 year-old woman who presented to the emergency service with non-specific abdominal discomfort, a palpable mass in left lower quadrant and constipation. She described a minor weight loss over the last 2 months. Her vital signs were normal and she was afebrile. Physical examination revealed a visible left lower quadrant mass that was tender and fixed. Rectal and gynaecological examinations were normal. Laboratory results were unremarkable, apart from a WBC of $14,600 / \mathrm{mm}^{3}$. Based on the findings and the state of acute abdomen, operative intervention was planned for the patient.

A median laparotomy was performed. At surgical exploration, a large firm mass surrounded by desmoplastic reaction was found in the intestines and was densely adherent to the 

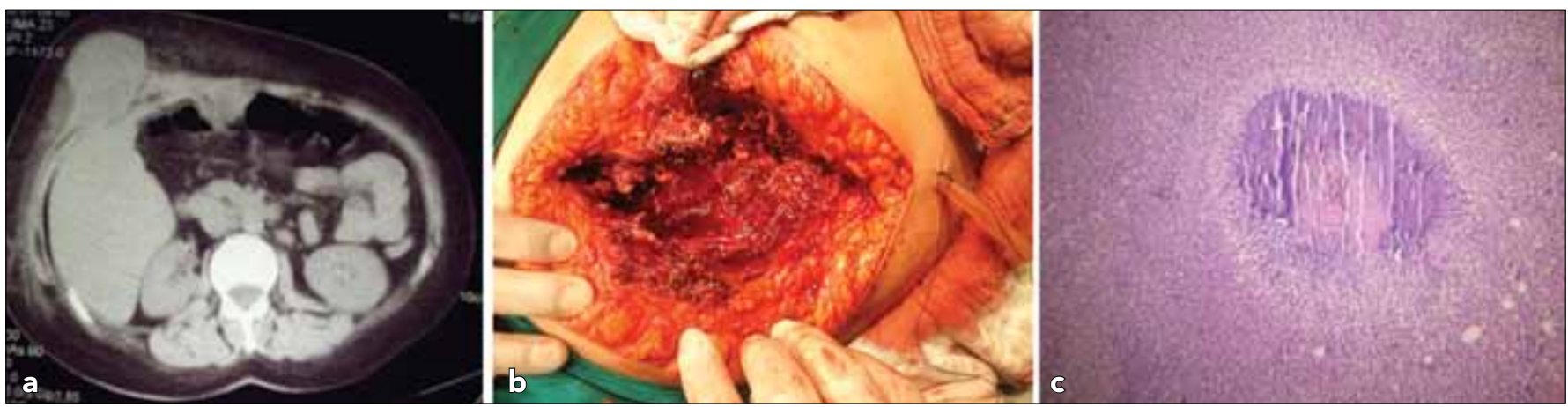

Figure 1. a-c. Abdominal CT scan shows a mass projecting internally from the right anterior abdominal wall (a), operative findings of primary actinomycosis of abdominal wall (b), H\&E staining X 100 (c). The image shows an actinomycotic abscess, an inflammatory infiltration composed largely of neutrophils.

anterior abdominal wall in the left lower quadrant. Total excision of the mass was performed including the involved lateral and transverse oblique muscles as well as the subcutaneous tissue and the adjacent jejunal loop. The resulting defect of the abdominal wall was closed with a $20 \times 20 \mathrm{~cm}$ prosthesis of vicryl mesh (Vicryl Mesh Knitted; (C) Ethicon, Inc., USA) Postoperatively, the patient recovered well without any complications. Histopathological diagnosis revealed actinomycosis with typical sulphur granules in the specimen. Medical treatment was commenced accordingly.

\section{Case 3}

An 18 year-old girl was referred to our clinic with a complaint of abdominal pain for 8 hours, with nausea, but no vomiting. There was right lower quadrant (RLQ) pain and tenderness with signs of peritonitis. Her medical history and blood investigations was unremarkable apart from a WBC of 15,200/ $\mathrm{mm}^{3}$. Abdominal US demonstrated a target sign in the RLQ measuring $3.5 \mathrm{~cm}$ on cross-section. Operation was planned with the suspect of acute appendicitis. Intraoperatively, the appendix was oedematous without signs of perforation. Macroscopic examination revealed a massively swollen but intact appendiceal wall.

Thereafter, the diagnosis of AMC of the appendix was established histologically. Microscopic examination revealed typical sulphur granules and small superficial sinuses in the appendiceal lumen (Figure 2). Postoperative antibiotic treatment consisted of high doses of penicillin IV for 4 weeks. Eight months after the operation, there were no complications.

\section{Discussion}

Actinomycosis is a clinical infection caused by Actinomyces israelii. The organism is normally found in the mouth, upper respiratory, gastrointestinal, anal and genital tracts. Actinomyces israelii has low virulence potential and does not cause disease unless the normal mucosal barrier is broken (3). When this occurs, it leads to multiple abscess formation, fistula or mass lesions (4). It most commonly affects the head and neck (50\%); however, almost any organ system can be involved (5). The abdomen can also be a site of presentation in nearly $20 \%$ of all patients. The appendix vermiformis and the iliocaecal region are the most common localisations of AMC.

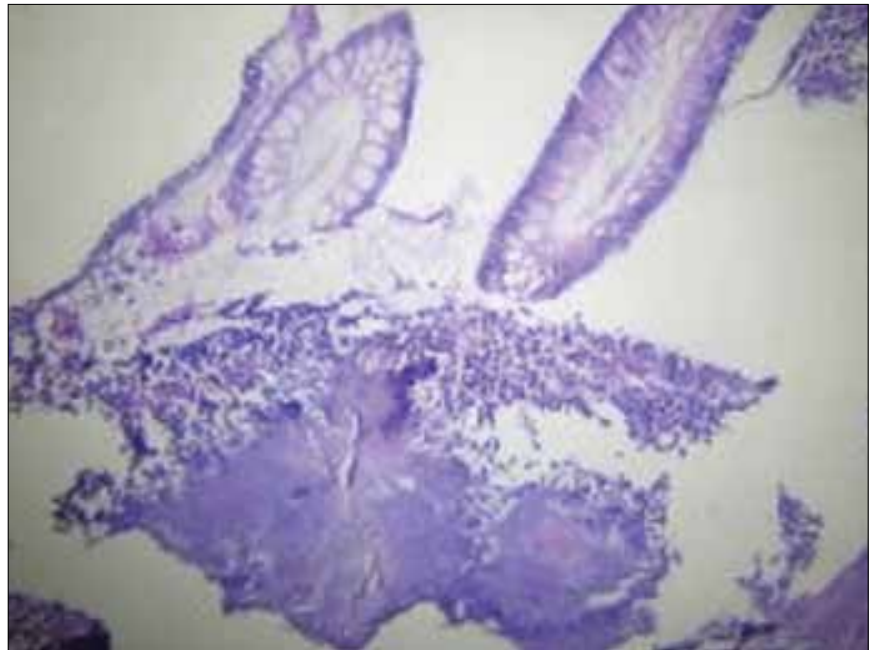

Figure 2. H\&E staining $X$ 200. Photomicrograph showing the actinomycotic colony within the lumen of the appendix.

The intestines, stomach, liver, pancreas, anorectal region, pelvis, or abdominal wall may also be involved. The clinical outcomes of abdominal AMC may present with nonspecific symptoms including abdominal pain, nausea, vomiting, fever, weight loss, and a palpable mass. Risk factors for abdominal $\mathrm{AMC}$ are recent abdominal operations or trauma, endoscopic procedures, the presence of intra-abdominal malignancy, and hollow viscous perforations (5). In women of child-bearing age, intrauterine contraceptive devices have been suggested as the primary colonisation site of $A M C$ in pelvis involvement (5). Any age group can be affected by AMC and male patients are affected more commonly than female patients, with a reported ratio of three to one. The reported annual incidence of actinomycosis is $1 / 300,000$ (6).

Isolated involvement of the abdominal wall is extremely rare. Similarly, there are only a few reports of clinical acute appendicitis due to AMC in the English literature (3). Laboratory tests and radiological imaging cannot significantly differentiate between AMC and other inflammatory or neoplastic processes. Preoperative diagnosis has been reported in only $10 \%$ of all cases (5). The differential diagnosis includes malignancies, amoeboma, inflammatory bowel diseases and abdominal muscle pathologies (5). 
Diagnosis is revealed by histopathological demonstration of the characteristic sulphur granules containing filaments in $50 \%$ of the cases. These findings, however, are not pathognomonic, because other organisms such as Nocardia and Streptomyces may aggregate sulphur granules resembling those found in Actinomycetes infections $(7,8)$. Microbiological isolation of the bacterium from bacterial cultures is usually inconclusive (9). Negative culture rates were reported to be as high as $76 \%(10)$.

In most cases, the diagnosis is often possible after surgical exploration and therefore the treatment becomes complex (9). The treatment of AMC is surgical drainage at the primary site of infection and, if a soft tissue tumor is suspected, a wide primary resection including the surrounding tissue. Following the confirmation of diagnosis, the systemic application of a high dose intravenous Penicillin (Penicillin G 10-20 million units daily) is the initial therapy of choice. After i.v. treatment for 4-6 weeks, oral penicillin (2-4 g/day) or amoxicillin should be administered for 6-12 months (11).

The rate of appendiceal localisation of AMC is about 65\%; furthermore, the incidence seems to be increasing (12). AMC may cause acute or chronic inflammation in the appendix $(12,13)$. The infectious process is generally limited (12), and haematogenous or lymphatic dissemination is usually rare. A resected appendix infected by Actinomyces shows typical sinuses in microscopic examination (13); as a rule, however, the clinical presentation cannot be differentiated from suppurative appendicitis $(12,13)$. Life-threatening complications such as abscesses and sinuses of the abdominal wall may occur if an actinomycotic inflammation of the appendix is left untreated (12). In the third case reported here, the diagnosis of primary actinomycosis of the appendix was revealed after pathological examination.

Abdominopelvic AMC is usually related with a history of trauma, abdominal surgery or intrauterine contraceptive devices (13). However, contrary to previous reports, no predisposing factors could be identified in our cases. In accordance with the literature, diagnosis could be made by histopathological examination of the specimen in all of our patients. Microscopic examination of the infected tissue should include a search for typical appearances of sulphur granules and, if possible, must be confirmed in microbiological cultures when a clinical suspicion of AMC arises.

All anatomic structures of the abdomen can be colonised by Actinomyces Israelii. Preoperative diagnosis is difficult because of the low index of suspicion, unusual presentations, and difficulty in culturing. In the majority of cases, the combination of surgery and antibiotic treatment results in complete treatment.

\section{Ethics Committee Approval: N/A.}

Informed Consent: Written informed consent was obtained from patients.

\section{Peer-review: Externally peer-reviewed.}

Author contributions: Concept - F.K., E.M.; Design - M.Ö., K.D.; Supervision - F.K., M.O.; Resource -S.Ö., E.M.; Materials - S.Ö., F.K.; Data Collection\&/or Processing - F.K.,E.M.; Analysis\&/or Interpretation - M.Ö., K.D.; Literature Search - F.K.; Writing - F.K., M.Ö.; Critical Reviews -M.Ö.

Conflict of Interest: No conflict of interest was declared by the authors.

Financial Disclosure: No financial disclosure was declared by the authors

\section{References}

1. Harsch IA, Benninger J, Niedobitek G, Schindler G, Schneider HT, Hahn EG, et al. Abdominal actinomycosis: complication of endoscopic stenting in chronic pancreatitis? Endoscopy 2001;33:1065-9. [CrossRef]

2. Seock $Y L$, Ji HL, Seung JL, Cheol SL. Primary Abdominal Wall Actinomycosis. J Med Cases 2012;3:4-6.

3. Schmidt P, Koltai JL, Weltzien A. Actinomycosis of the appendix in childhood. Pediatr Surg Int 1999;15:63-5. [CrossRef]

4. Berardi RS. Abdominal actinomycosis. Surg Gynecol Obstet 1979;149:257-66.

5. Ladurner R, Bogner JR, Drosse I, Volkmer E, Sommerey S, Hohenbleicher $F$, et al. A rare case of primary actinomycosis of the anterior abdominal wall: diagnosis and treatment. Hernia 2008;12:549-52. [CrossRef]

6. Weese WC, Smith IM. A study of 57 cases of actinomycosis over a 36-year period. A diagnostic 'failure' with good prognosis after treatment. Arch Intern Med 1975;135:1562-8. [CrossRef]

7. Pitot D, De Moor V, Demetter P, Place S, Gelin M, El Nakadi I. Actinomycotic abscess of the anterior abdominal wall: a case report and literature review. Acta Chir Belg 2008;108:471-3.

8. Wagenlehner FM, Mohren B, Naber KG, Männl HF. Abdominal actinomycosis. Clin Microbiol Infect 2003;9:881-5. [CrossRef]

9. Acquaro P, Tagliabue F, Confalonieri G, Faccioli P, Costa M. Abdominal wall actinomycosis simulating a malignant neoplasm: Case report and review of the literature. World J Gastrointest Surg 2010;2:247-50. [CrossRef]

10. Hefny AF, Joshi S, Saadeldin YA, Fadlalla H, Abu-Zidan FM. Primary anterior abdominal wall actinomycosis.Singapore Med J 2006;47:419-21.

11. Hamid D, Baldauf JJ, Cuenin C, Ritter J. Treatment strategy for pelvic actinomycosis: case report and review of literature. Eur $J$ Obstet Gynecol Reprod Biol 2000;89:197-200. [CrossRef]

12. Nissotakis C, Sakorafas GH, Koureta T, Revelos K, Kassaras G, Peros G. Actinomycosis of the appendix: diagnostic and therapeutic considerations. Int J Infect Dis 2008;12:562-4. [CrossRef]

13. Yiğiter M, Kıyıcı H, Arda IS, Hiçsönmez A. Actinomycosis: a differential diagnosis for appendicitis. A case report and review of the literature. J Pediatr Surg 2007;42:E23-6. [CrossRef] 